\title{
4-dimensional, multiphase, steady-state imaging with contrast enhancement (MUSIC) in the heart; a feasibility study in children
}

\author{
Fei Han ${ }^{1,2^{*}}$, Stanislas Rapacchi ${ }^{1}$, Sarah N Khan ${ }^{1}$, Ihab Ayad ${ }^{3}$, Isidro Salusky ${ }^{4}$, Adam Plotnik', Simon Gabriel', \\ Paul J Finn ${ }^{1,5}$, Peng $\mathrm{Hu}^{1,5}$ \\ From 18th Annual SCMR Scientific Sessions \\ Nice, France. 4-7 February 2015
}

\section{Background}

Conventional breath-held gadolinium-based contrastenhanced MR angiography (CE-MRA) provides excellent definition of extra-cardiac anatomy, but does not provide diagnostic image quality for intra-cardiac anatomy because it is not gated to ECG due to time constraints associated with breath-holding and the need to capture the first-pass of gadolinium. To address this issue, we propose a 4D non-breath-held multiphase steady-state imaging sequence (MUSIC) using ferumoxytol, which is an FDA approved iron-oxide particle for treating irondeficiency anemia, as an intravascular contrast agent and demonstrate its feasibility in children with congenital heart disease (CHD).

\section{Methods}

Eight pediatric CHD patients underwent cardiac MRI under general anesthesia and mechanical ventilation, which is our standard practice for young children who cannot cooperate with breath-holding instructions. The breath-held CE-MRA was first performed under ventilator-controlled breath-hold (VCBH) during the first-pass of a ferumoxytol bolus injection $(4 \mathrm{mg}-\mathrm{Fe} / \mathrm{kg})$ and repeated 2-3 min later during the delayed-phase. Subsequently, the cardiac-phase-resolved 4D MUSIC acquisition (TR/TE= 2.9/0.9ms; $\mathrm{FA}=15^{\circ}$; isotropic resolution $=0.6-0.9 \mathrm{~mm}, 5-8$ cardiac phases, scan time 4-8min, 65-95ms temporal resolution) was performed during the steady-state distribution phase of ferumoxytol without VCBH and the ventilator tubing air pressure signal was fed into the MR scanner for respiratory gating. Standard 2D cardiac cine images were

${ }^{1}$ Radiological Sciences, David Geffen School of Medicine at UCLA, Los Angeles, CA, USA

Full list of author information is available at the end of the article also acquired under VCBH. Subjective image quality scores ( 1 =poor, $2=$ fair, $3=$ good, $4=$ =xcellent) were visually assessed and the LV volumes were measured based on standard 2D cine and the 4D MUSIC data.

\section{Results}

As shown in Fig. 1, compared to VCBH CE-MRA, the 4D MUSIC provides much improved definition of intracardiac structures (e.g. cardiac chambers, coronary arteries and the valves) by eliminating cardiac motion blurring. Fig.2a shows reformatted cardiac four-chamber view at select cardiac phases from which the $L V$ volumes could be calculated. In Fig. 2b, all three major branches of the coronary artery are clearly visualized by reformatting the 4D MUSIC acquired on an 8-monthold patient. In 7 out of the 8 patients, the origins and course of the coronary arteries, including 2 anomalous origins were identified based on the 4D MUSIC image. In one patient, the 4D MUSIC approach pre-empted an invasive diagnostic catheterization. 4D MUSIC provided significantly better image scores for intra-cardiac anatomy than the first-pass MRA (3.6 \pm 0.5 vs. $2.0 \pm 0.4$ $\mathrm{P}<0.05)$. The LV volume measurements based on $2 \mathrm{D}$ cine and 4D MUSIC correlated well (concordance correlation coefficient $>0.95$ ).

\section{Conclusions}

Our study represents a potential new paradigm of cardiovascular MRI for young children. The use of ferumoxytol (14 hours of intravascular half-life) eliminates the time constraints of conventional CE-MRA and enables much higher quality 4D dynamic cardiac imaging with sub-millimeter isotropic resolution. 


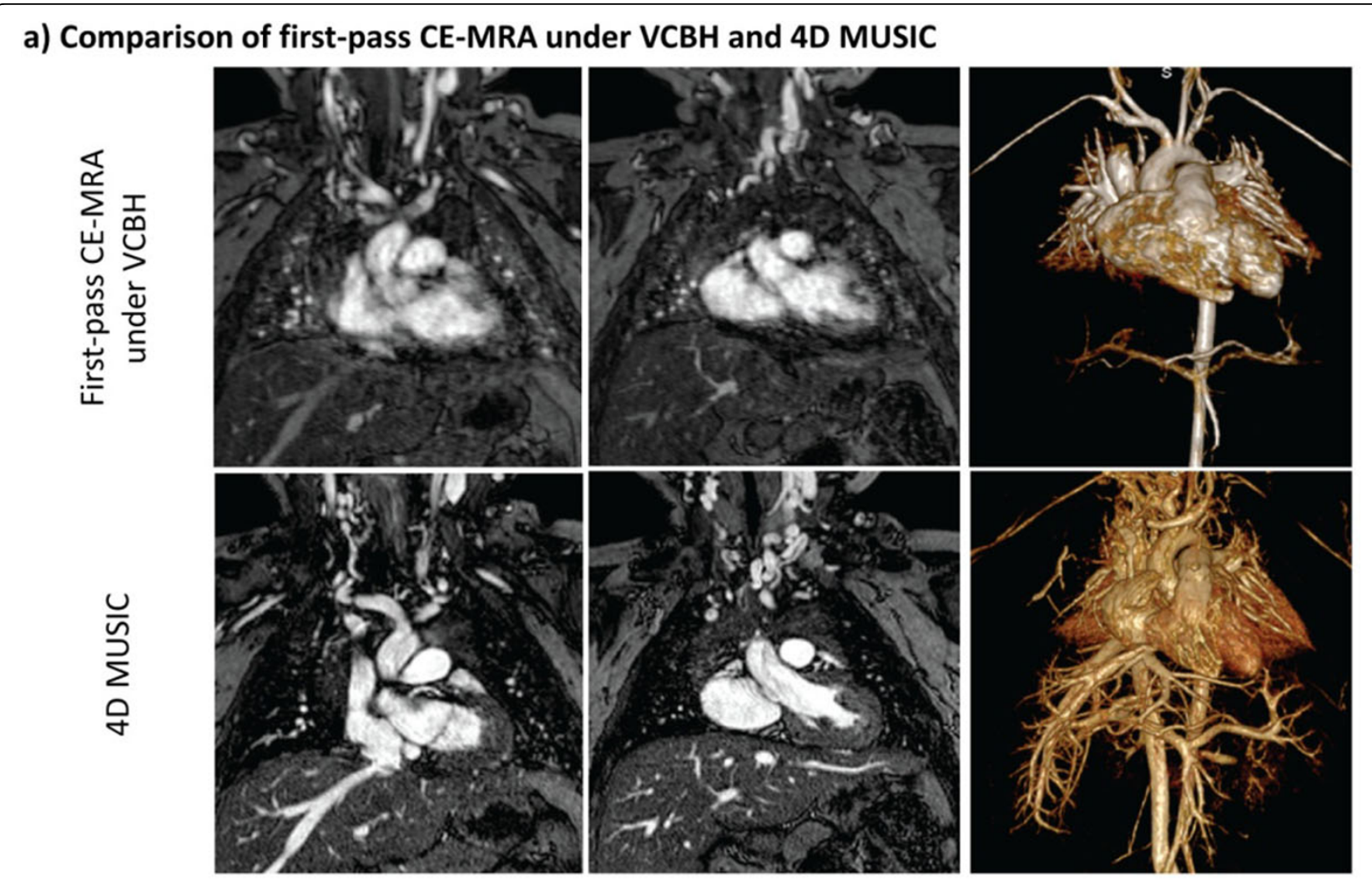

b) Comparison of the CE-MRA under VCBH (first-pass and delayed-phase) and the 4D MUSIC

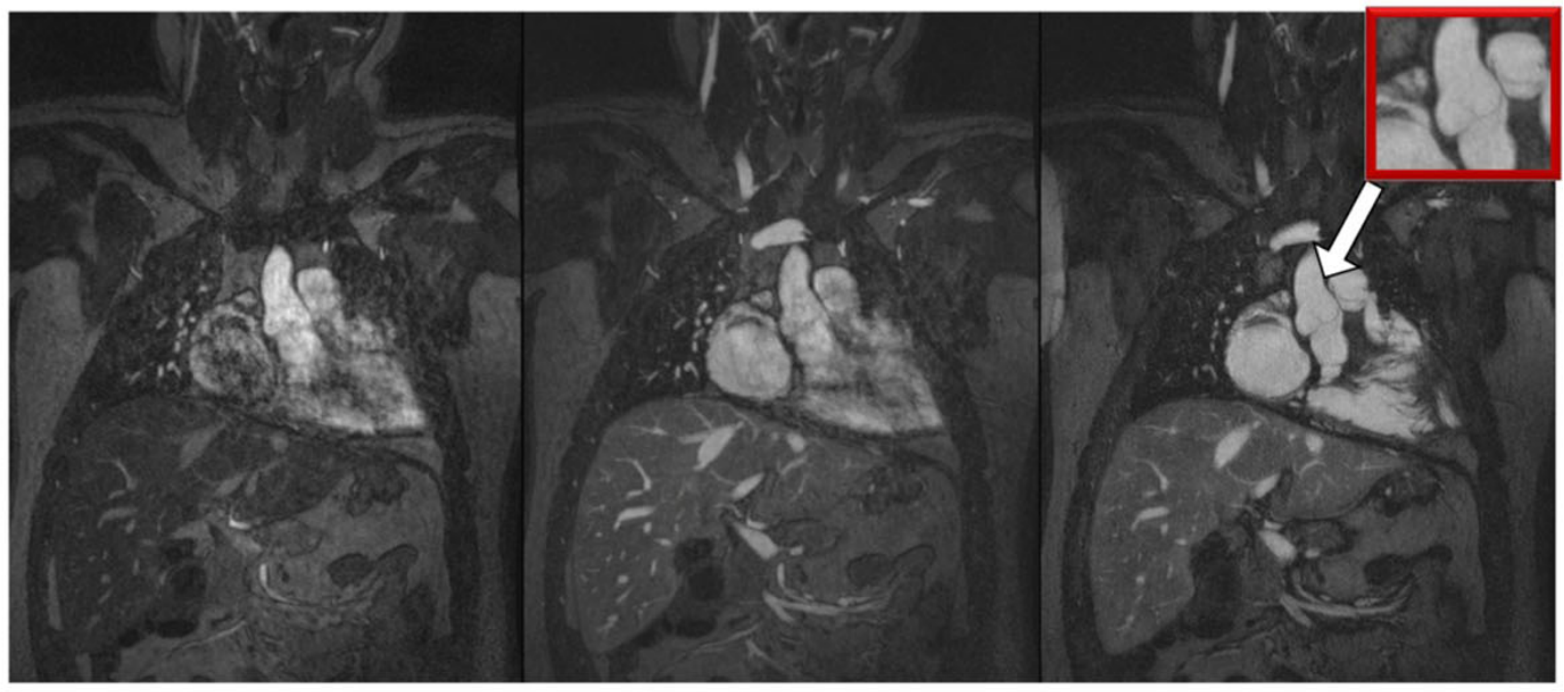

First-pass CE-MRA under $\mathrm{VCBH}$
Delayed-phase CE-MRA under $\mathrm{VCBH}$
4D MUSIC

Figure 1 a) Selected 2 slices and 3D rendering of first-pass CE-MRA under VCBH and the 4D MUSIC (phase \#3 is chosen out of 5 cardiac phases for display) on a 4 y.o. 10kg boy. The structures in and close to the heart (including the cardiac chambers, coronary arteries, aortic root, main pulmonary artery, and valves) are much better defined using 4D MUSIC. b) Comparison of the first-pass VCBH CE-MRA, delayed phase VCBH CEMRA, and the proposed 4D MUSIC. The 4D MUSIC image provided excellent definition of cardiac anatomy including the aortic cusps and the aortic valve leaflets (red box), and the trabeculations within the cardiac chambers, while both first-pass and delayed-phase VCBH CE-MRA suffers from significant cardiac motion blurring. Signal intensity of the 4D MUSIC image is uniform across the entire FOV. 


\section{a) Selected cardiac phases of 4D MUSIC}

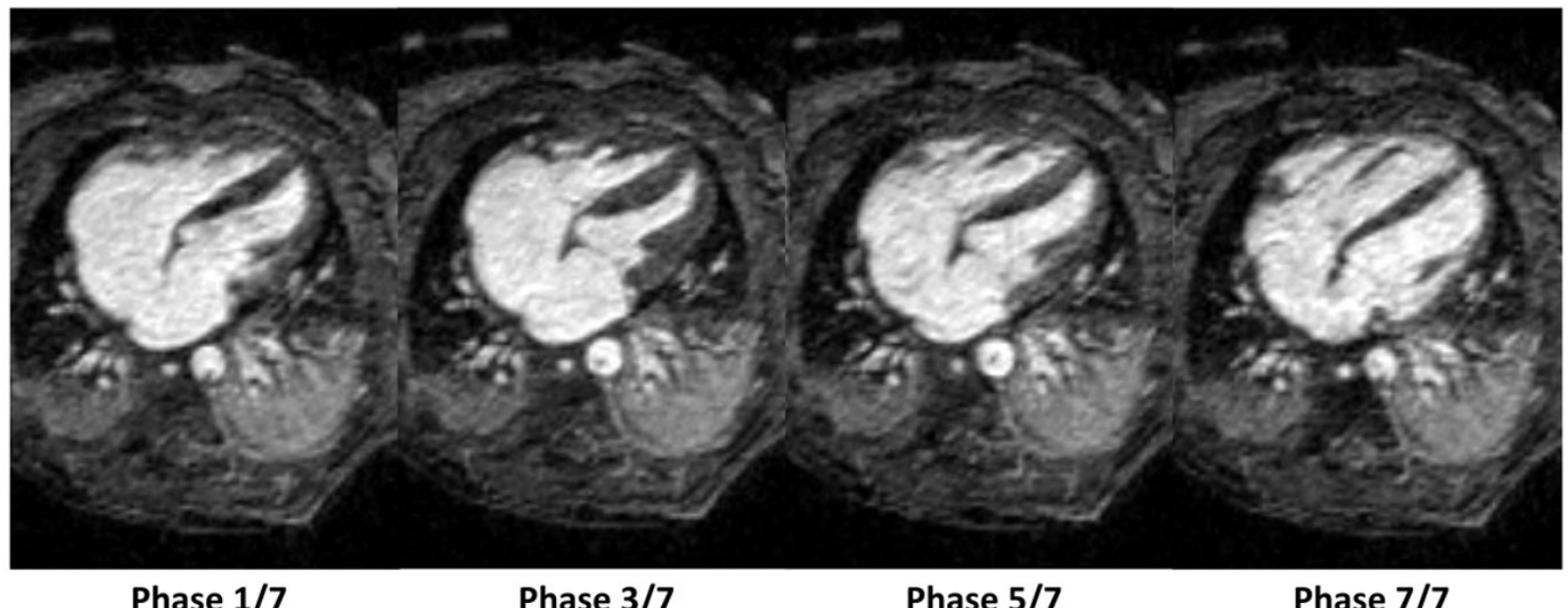

\section{b) Reformatted 4D MUSIC data for coronary artery visualization}

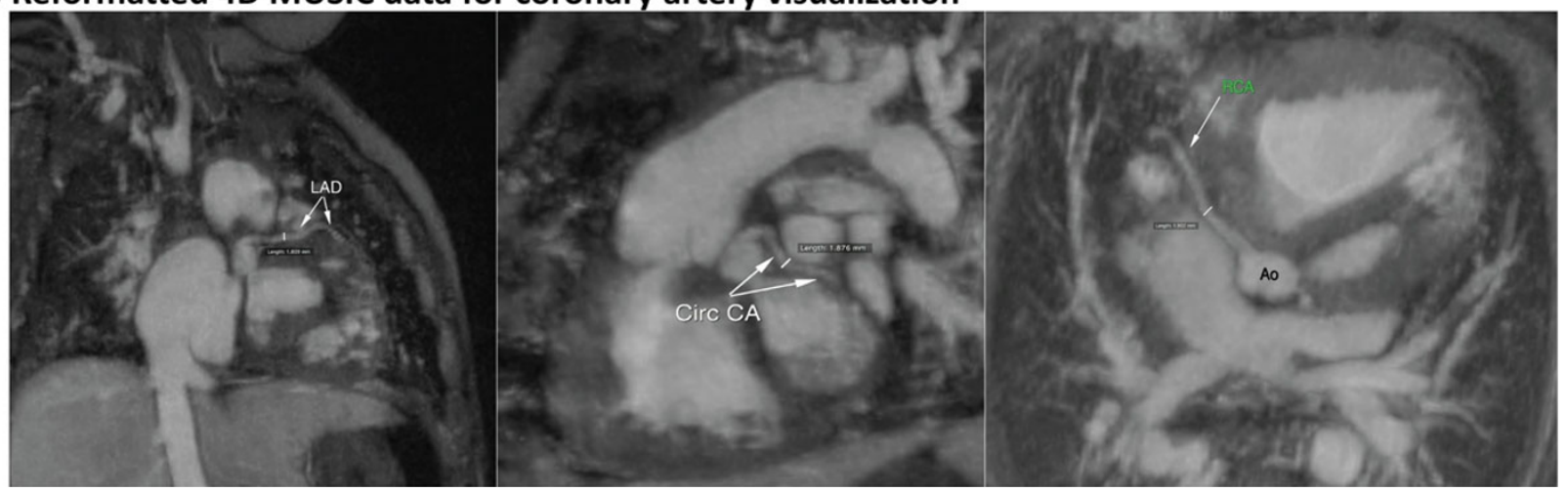

Figure 2 a) Reformatted cardiac four chamber view images on selected cardiac phases (4 out of 7) based on the 4D MUSIC on a 3 d.o. 2kg boy. No 2D cine was acquired on this patient due to concerns of cardiopulmonary inefficiency and clinical assessment of cardiac function and volumes was based on the 4D MUSIC data. b) All three major branches of the coronary artery (LAD: left anterior descending; Circ CA: left circumflex; RCA: right coronary arteries) are clearly visualized by reformatting the 4D MUSIC data acquired in an 8-month-old 7kg boy with complex CHD. The multiphase data have $0.9 \mathrm{~mm}$ isotropic resolution.

\section{Funding}

NIH (NHLBI) 1R21 HL113427.

\section{Authors' details}

${ }^{1}$ Radiological Sciences, David Geffen School of Medicine at UCLA, Los Angeles, CA, USA. ²Department of Bioengineering, University of California, Los Angeles, Los Angeles, CA, USA. ${ }^{3}$ Department of Anesthesiology, David Geffen School of Medicine at UCLA, Los Angeles, CA, USA. ${ }^{4}$ Department of Pediatrics, David Geffen School of Medicine at UCLA, Los Angeles, CA, USA. ${ }^{5}$ Biomedical Physics Inter-Departmental Graduate Program, University of California, Los Angeles, Los Angeles, CA, USA.

\section{Published: 3 February 2015}

\section{doi:10.1186/1532-429X-17-S1-Q131}

Cite this article as: Han et al:: 4-dimensional, multiphase, steady-state imaging with contrast enhancement (MUSIC) in the heart; a feasibility study in children. Journal of Cardiovascular Magnetic Resonance 201517 (Suppl 1):Q131.
Submit your next manuscript to BioMed Central and take full advantage of:

- Convenient online submission

- Thorough peer review

- No space constraints or color figure charges

- Immediate publication on acceptance

- Inclusion in PubMed, CAS, Scopus and Google Scholar

- Research which is freely available for redistribution 